\title{
Theorems on polynomials in right invertible operators
}

\section{Przeworska-Rolewtoz}

Sei $D$ ein rechtsinvertierbarer- Operator in einem linearen Raum $X$ und $Q(D)$ ein Polynom von $D$ mit Operatorkoeffizienten. Es wird gezeigt, da $B$ unter gewissen Bedingungen auf $\boldsymbol{X}$ für die Rechtsinverse $R$ und die Koeffizienten des Polynoms $Q(D)$ genau dann $Q(D)=0$ gilt, fälls $Q(D) R^{k} z=0(k=0,1,2, \ldots)$ für alle $z$ aus ker $D$.

Предположим, что $D$ - право обратимый оператор, действующиґ в линейном пространстве $X$ и $Q(D)$ - многочлен с операторными коеффициентами. В этом случае $Q(D)=0$ тогда и только тогда, когда $Q(D) R^{k_{z}}=0$ для всех $z \in \operatorname{ker} D(k=0,1,2, \ldots)$ если выполнены некоторые условия на $X$, правы обратный $R$ и коеффицкенты многочлена $Q(D)$.

Suppose that $Q(D)$ is a polynomial in a right invertible operator acting in a linear space $X$, in general, with operator coefficients. Then $Q(D)=0$ if and only if $Q(D) R^{k} z=0$ for all $z \in \operatorname{ker} D(k=0,1,2, \ldots)$ under appropriate assumptions on $X$, the right inverse $R$ of $D$ and coefficients of $Q(D)$.

Iet $\boldsymbol{P}_{1}(D), \boldsymbol{P}_{2}(D)$ be polynomial differential operators, i.e. finite sums of monomials of the form

$$
A_{0}(x) D A_{1}(x) \ldots A_{n-1}(x) D A_{n}(x) \quad \text { where } \quad D=\frac{d}{d t}
$$

and $A_{f}(x)$ are given differentiable functions. Using the identity

$$
D A_{j}(x)=A_{j}(x) D+A_{j}{ }^{\prime}(x)
$$

we can rewrite these polynomials in the form:

$$
P_{i}(D)=\sum_{j=1}^{m} P_{j}^{(i)}(x) D^{j} \quad(i=1,2)
$$

The following theorem holds for polynomial differential operators.

Theorem 1 (cf. CARutT [1, 2], Ince [3]): Suppose that $P_{1}(D)$ and $P_{2}(D)$ are two polynomial differential operators. Then

$$
P_{1}(D)=P_{2}(D)
$$

if and only if

$$
P_{1}(D) x^{k}=P_{2}(D) x^{k} \quad \text { for } \quad k=0,1,2, \ldots
$$

In particular, suppose that $P(D)$ is a polynomial differential operator. Then

$$
P(D)=0
$$

if and only if

$$
P(D) x^{k}=0 \quad(k=0,1,2, \ldots) .
$$


In order to prove (2) it is enough to prove (3).

This result can be generalized for arbitrary right invertible operators, in particular, for difference operators (cf. [2]). Namely, suppose that $X$ is a linear space over a field $\mathscr{F}$ of scalars, $D$ is a right invertible operator and $\operatorname{dim} \operatorname{ker} D>0$. If $R$ is an arbitrarily fixed right inverse of $D$ then elements of the form $R^{k} z_{k}$, where $z_{k} \in \operatorname{ker} D$ and $k=0,1,2, \ldots$, play a role of monomials for $D$ and are linearly independent (cf. [4]). The set

$$
P(R)=\operatorname{lin}\left\{R^{k} z: z \in \operatorname{ker} D \quad(k=0,1,2, \ldots)\right\}
$$

is independent of the choice of a right inverse $R$.

Now, consider 3 different cases:

Theorem 2: Let

$$
q(D)=\sum_{j=0}^{n} q_{j} D^{j}
$$

be an arbitrary polynomial with scalar coefficients. Then $q(D)=0$ if and only if

$$
q(D) R^{k} z=0 \text { for all } z \in \operatorname{ker} D \quad(k=0,1,2, \ldots)
$$

where $R$ is an arbitrarily fixed right inverse of $D$.

Proof: Necessity is obvious. In order to prove that the condition (4) implies $q(D)=0$ observe that $R^{k} z \in \operatorname{ker} D^{k+1}(k=0,1,2, \ldots)$ for all $z \in \operatorname{ker} D$ (cf. also [4]). Then for all $z \in \operatorname{ker} D(k=0,1,2, \ldots)$ we find

$$
\begin{aligned}
0 & =f(D) R^{k} z=\sum_{j=0}^{n} q_{j} D^{i} R^{k} z \\
& =\sum_{j=0}^{k} q_{j} R^{k-j_{z}}+\sum_{j=k+1}^{n} q_{j} D^{j-k}=\sum_{j=0}^{k} q_{j} R^{k-j_{z}}
\end{aligned}
$$

since, by definition, $D R=I$ and $D z=0$. This, and the linear independence of elements $z, R z, R^{2} z, \ldots$ together imply that $q_{0}, \ldots, q_{k}=0$ for $k=0,1,2, \ldots, n$, i.e. $q(D)=0$

Observe that in Theorem 2 it is sufficient to admit only a finite number of conditions of the form (5). Namely, we obtain the same result if $k=0,1,2, \ldots, m$ where $m=n \cdot \operatorname{dim} \operatorname{ker} D+1$.

Theorem 3: Suppose that $X$ is a D-algebra, i.e. a commutative linear ring (with a non-trivial multiplication) such that

$$
\text { if } x, y \in \text { dom } D \text { then } x y \in d o m D \text {. }
$$

Suppose, moreover, that ker $D$ is not an annihilator in $X$, i.e. if $x$ ker $D=\{0\}$ for an $x \in X$ then $x=0$. Let

$$
q(D)=\sum_{k=0}^{n} q_{k} D^{k}, \text { where } q_{0}, \ldots, q_{n} \in X
$$

Then $q(D)=0$ if and only if

$$
q(D) R^{k} z=0 \text { for all } z \in \operatorname{ker} D \quad(k=0,1,2, \ldots)
$$

where $R$ is an arbitrarily fixed right inverse of $D$.

Proof: Necessity is obvious. Sufficiency will be proved by induction. Assume that the condition (8) holds. In a similar way, was in the proof of Theorem (8) we shall 
rewrite (8) in the form

$$
\sum_{j=0}^{k} q_{j} R^{k-j} z=0 \text { for all } z \operatorname{ker} D \quad(k=0,1,2, \ldots) .
$$

Let $k=0$. Then we have $q_{0} R^{k} z=0$ and $R^{k} z \neq 0$ for $z=0$ (because if $R u=0$ then $u=D R u=0$ ). The arbitrariness of $z \in \operatorname{ker} D$ and our assumptions together imply that $q_{0}=0$. Suppose that $q_{0}=\cdots=q_{m}=0$ for an arbitrarily fixed $m \geqq 0$. Then

$$
0=\sum_{j=0}^{n+1} q_{j} R^{m+1-i} z=q_{m+1} z
$$

The arbitrariness of $z$ ker $D$ implies $q_{m+1}=0$, which finishes the proof

Theorem 4: Suppose that $X$ is a complete linear metric space, $R$ is an arbitrarily fixed right inverse of $D$ and

$$
Q(D)=\sum_{k=0}^{n} Q_{k} D^{k}
$$

where $Q_{k}$ : dom $D^{n} \rightarrow X$ are arbitrary linear operators. Suppose, moreover, that the operator $Q(D)$ is closed, $P(R) \subset \operatorname{ker} Q(D)$ and $\overline{P(R})=X$. Then $Q(D)=0$ if and only if

$$
Q(D) R^{k} z=0 \quad \text { for all } z \in \operatorname{ker} D \quad(k=0,1,2, \ldots) \text {. }
$$

Proof: Necessity is obvious. Sufficiency follows from the fact that by our assumptions we have

$$
X=\overline{P(R)} \subset \overline{\operatorname{ker} Q(D)}=\operatorname{ker} Q(D) .
$$

This implies $Q(D)=0$

Note that from proofs of Theorems 2 and 3 follows that

$$
P(R) \subset \operatorname{ker} Q(D)
$$

which is assumed in Theorem 4.

\section{REFERENCES}

[1] Carditz, L.: Some Operational Formulas. Math. Nachrichten 45 (1970), 379-389.

[2] Carlitz, L.: A Theorem on Differential Operators. Amer. Math. Monthly 83 (1976), 351 bis 354 .

[3] Inoe, E. L.: Ordinary Differential Equations, London 1927.

[4] Przeworska-Rolewicz, D.: Algebraic Theory of Right Invertible Operators. Studia Math. 48 (1973), $129-143$.

[5] Przeworska-Rolewicz, D.: Shifts and Periodicity for Right Invertible Operators. Research Notes in Mathematics 43, Pitman Advanced Publish. Program. Boston-London-Melbourne 1980 .

Manuskripteingang: 20. 2. 1981

\section{VERFASSER :}

Prof. Dr. Danuta Przeworska-Rolewicz

Instytut Matematyczny PAN

P-00-950 Warszawa, skr. pocztowa 137 